\title{
Integration of Adaptive Process Control with Computational Simulation for Spin-Forming
}

\author{
Peter I. Raboin \\ and \\ Anthony S. Lee
}

March 10, 1998

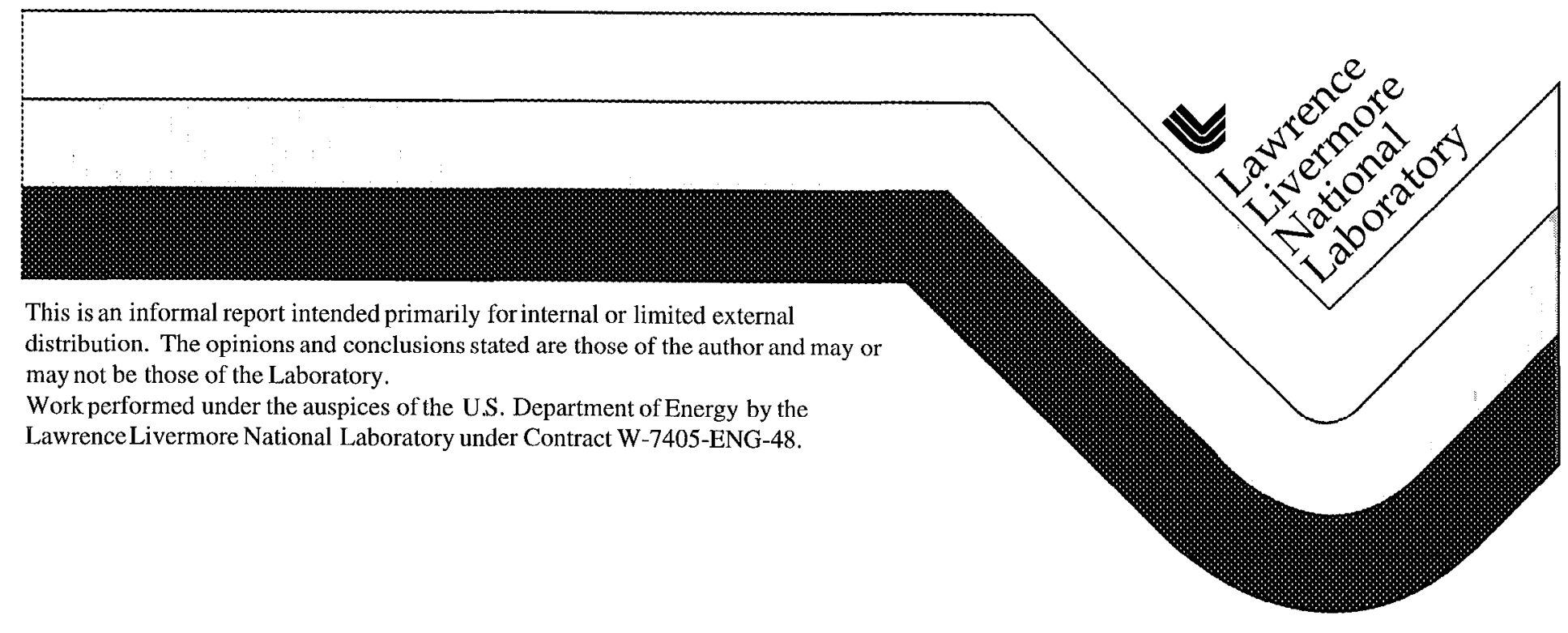




\section{DISCLAIMER}

This document was prepared as an account of work sponsored by an agency of the United States Government Neither the United States Government nor the University of California nor any of their employees, makes any warranty, express or implied, or assumes any legal liability or responsibility for the accuracy, completeness, or usefulness of any information, apparatus, product, or process disclosed, or represents that its use would not infringe privately owned rights. Reference herein to any specific commercial product, process, or service by trade name, trademark, manufacturer, or otherwise, does not necessarily constitute or imply its endorsement, recommendation, or favoring by the United States Government or the University of California. The views and opinions of authors expressed herein do not necessarily state or reflect those of the United States Government or the University of California, and shall not be used for advertising or product endorsement purposes

This report has been reproduced directly from the best available copy.

Available to DOE and DOE contractors from the Office of Scientific and Technical Information

PO Bux 62, Oak Ridge, TN 37831

Prices available from (423) 576-8401

Available to the public from the National Technical Information Service

US Department of Commerce

5285 Port Royal Rd,

Springfield, VA 22161 


\title{
Integration of Adaptive Process Control with Computational Simulation for Spin-Forming
}

\author{
by \\ Peter J. Raboin \\ and \\ Anthony S. Lee \\ The University of California \\ Lawrence Livermore National Laboratory \\ P.O. Box 808 \\ Livermore, CA 94550 USA
}

Keywords: metal forming, finite element, shape-memory

\begin{abstract}
Improvements in spin-forming capabilities through upgrades to a metrology and machine control system and advances in numerical simulation techniques were studied in a two year project funded by Laboratory Directed Research and Development (LDRD) at Lawrence Livermore National Laboratory. Numerical analyses were benchmarked with spin-forming experiments and computational speeds increased sufficiently to now permit actual part forming simulations. Extensive modeling activities examined the simulation speeds and capabilities of several metal forming computer codes for modeling flat plate and cylindrical spin-forming geometries. Shape memory research created the first numerical model to describe this highly unusual deformation behavior in Uranium alloys. A spin-forming metrology assessment led to sensor and data acquisition improvements that will facilitate future process accuracy enhancements, such as a metrology frame. Finally, software improvements (SmartCAM) to the manufacturing process numerically integrate the part models to the spin-forming process and to computational simulations.
\end{abstract}




\section{Introduction}

Spin-forming is a metal forming operation where the starting blank material (a plate) is pressed between a Mandrel and Tail-Stock. The entire assembly revolves in a fashion similar to a pottery wheel (Figure 1). Two rotating tools force the work-piece onto the mandrel causing permanent plastic deformation. The tool motion, rotation speeds, number of tool passes, mandrel shape and workpiece dimensions are all forming process parameters which are varied to produce a desired final part shape with the acceptable part characteristics, such as residual stresses and strains.

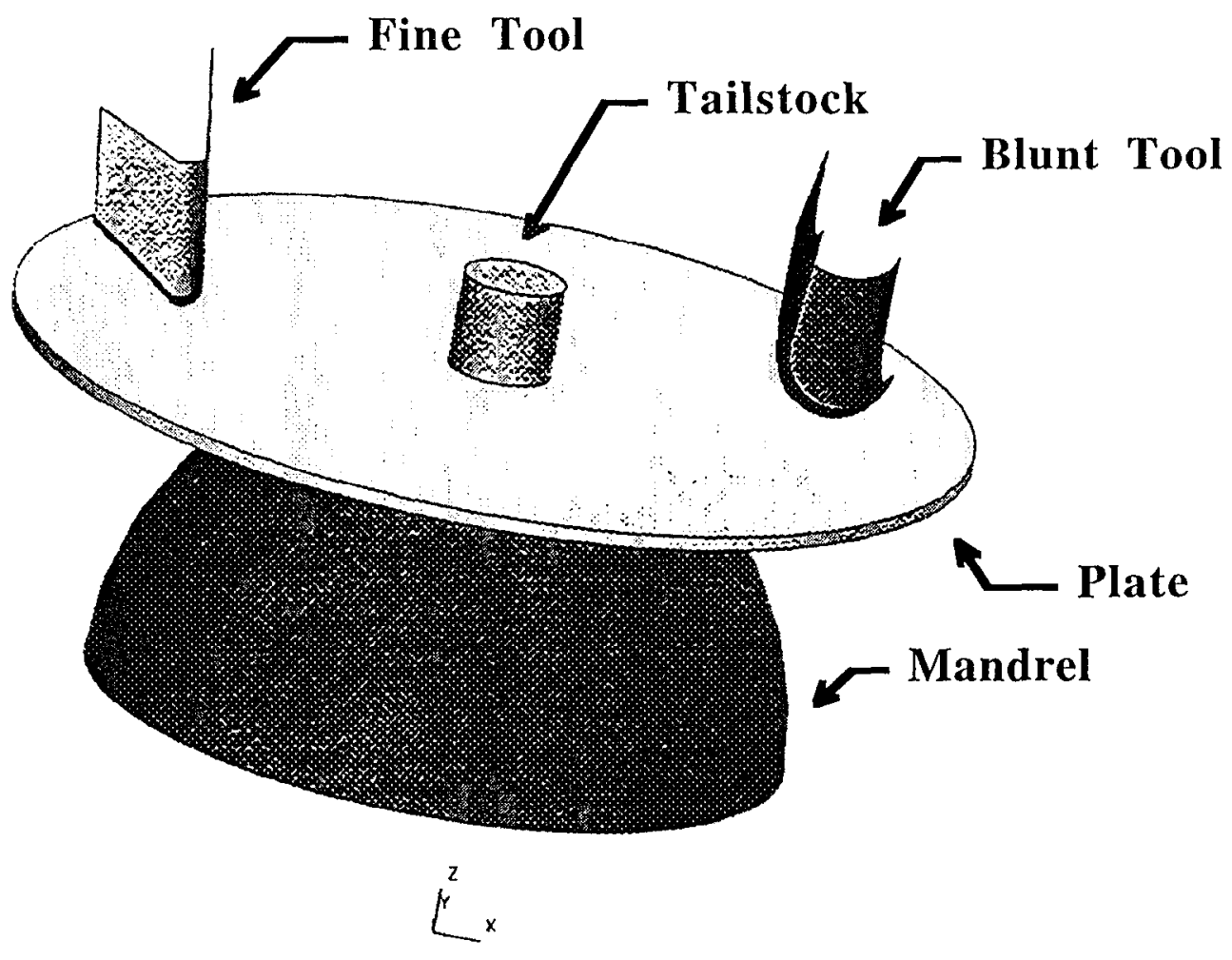

Figure 1 Flat plate spin-forming parts

Spin-forming is seen as one manufacturing method for achieving a low volume and cost effective production capability for the DOE/DP complex. Industrial spin-forming applications are typically high volume and therefore low cost operations. The key feature of spin-forming which is of interest to the DOE/DP is its reproducibility, and the fact that spin-formed parts achieve a degree of wrought like properties through work hardening. Once a spinforming process is defined, the production costs are typically lower than methods which require a forging press or other expensive tooling.

To establish spin-forming as a viable manufacturing option, improvements in forming accuracy/control and a better predictive understanding of the physical processes involved in spin-forming are needed. Integrating real time process information to monitor and/or control the forming operation is the biggest step in defining a reproducible production process. Realistic computational simulations can determine the effects of varying the forming process parameters. Through simulations, an understanding of the spin-forming process phenomena can be finally achieved.

This LDRD project has had four major activities aimed towards improving the quality of spin-forming at LLNL. The first was to integrate the numerical controls with three dimensional (3D) spin-forming analyses. The second item was to install a metrology system to improve machine accuracy. Third was to complete some analysis objectives: benchmarking code predictions, model cylindrical geometry's and increase simulation speeds through 
the use of parallel code calculations. The last activity was to improve the Uranium constitutive model by modeling shape memory effects.

\section{Numerical System Integration}

Integrating the part models to the actual machine profile programming was achieved this year through a Computer Aided Manufacturing (CAM) package called SmartCAM. The software was installed on the spin-forming PC which also serves as the data acquisition system. The software was also installed on a SUN workstation where designers and drafters have access to the original CAD models. SmartCAM operator training was also completed. SmartCAM has successfully taken old part files and provided spin-forming G-code programs. The G-code files are machine control instructions which define the tool movement and spin-forming process. The software and data acquisition system now provide the necessary information for integration with numerical simulations.

\section{Spin-Forming Metrology}

Metrology measurements made on the spin-forming machine to identify the sources of machine errors and to indicate how to develop possible methods to reduce these errors. As a result of metrology tests of the spinforming machine, several significant findings are reportable;

- Static errors sources such as the displacement vs. commanded position are repeatable and measurable.

- Considering the forces applied (tests were conducted up to 15,000 lbs. of force), the machine displacement is remarkably repeatable. This means that if the forces can be measured either directly or indirectly (pressure transducers) and it is possible to predict and compensate for force induced displacement errors.

- The following error as a function of machine velocity is linear and measurable and repeatable. It is also approaches no-load levels even with extremely high forces applied to the slides.

These reports are good news and they are probably due to the hydraulics not reflecting back into the control system in a manner similar to a typical electric motor - drive configuration.

The design and construction of a metrology frame that would use sensors and a computer to correct for machine errors in real time was not completed as planned for this project. A delay in the second year funding start and over-riding programmatic priorities diverted designers from completing this task. Funding for this activity was returned to LDRD. A data acquisition system was added to the spin-forming system to generate reliable data at an update rate that would be able provide useful control information. The pressure, displacement and CNC following error are now readable and additional sensor data is also available. The data acquisition system is expandable when such a need arises.

\section{Spin-Forming Experiments}

Benchmarking spin-forming experiments and calculations were carried out using a problem which is much simplified from the complicated programs used to form hemispherical parts. The two major simplifications were to use 304 Stainless Steel and to execute a straight downward plunge of the spinning tools. Thus, the tool motion was only along the spinning axis and there was no radial motion. The purpose of these experiments was to create some experimental data to measure the accuracy of spin-forming calculations, and to create a simple problem that would facilitate computational performance benchmarking.

A flat circular plate of 304 Stainless Steel of 1/4 inch thickness and 18 inch diameter was pressed onto a spherical mandrel of 12 inch diameter. The spinning tools were each located at a 6 inch radius. The spinning tools themselves are made from A2 Hardened Tool Steel. They each have a major rolling diameter of $10^{\prime}$. They have different rim contact radii of $3 / 8$ and 1 inch respectively. The differing tool contact radii cause distinct deformation patterns on the spin-formed part surfaces. The smaller radius is intended to provide more detailed forming contours while the larger radius tends to smooth the deformation over a larger area. There are separate Gcode instructions for each tool, and these instructions take into account the differing tool radii.

The tool motion for these benchmarking experiments consists of a simple downward movement of between 25.4 and $38.1 \mathrm{~mm}$. The spinning speed was varied between 3 and 6 revolutions/sec and the tool speed varied between 100 and $200 \mathrm{~mm} / \mathrm{min}$. Table 1 shows three results for a $38.1 \mathrm{~mm}$ tool movement and the amount of permanent deformation left in the work piece. The $\Delta$ displacement is the dome height under the finished plate and except for 
the effects of plate thinning which was observed, the $\Delta$ displacement could have been as high as $38.1 \mathrm{~mm}$. The difference between $38.1 \mathrm{~mm}$ and $\Delta$ is the springback for the purpose of discussion in this report. A more detailed set of part measurements (sectioning) is needed to account for part thinning and accurately define the springback quantity.

\begin{tabular}{|c|c|c|c|c|}
\hline Run & $\begin{array}{c}\text { Spin Speed } \\
(\mathrm{rev} / \mathrm{sec})\end{array}$ & $\begin{array}{c}\text { Tool Speed } \\
(\mathrm{mm} / \mathrm{min})\end{array}$ & $\Delta(\mathrm{mm})$ & $\begin{array}{c}\text { Springback } \\
(\mathrm{mm})\end{array}$ \\
\hline 1 & 3 & 100 & 28.85 & 9.25 \\
\hline 2 & 6 & 200 & 28.30 & 9.80 \\
\hline 3 & 6 & 100 & 29.97 & 8.13 \\
\hline
\end{tabular}

Table 1 Flat plate experiment springback results

These experimental results demonstrate the important effect of tool feed rates on spinning. Even though the tools pressed the plates down $38.1 \mathrm{~mm}$ in each run, the amount of springback varied due to the process parameters of spin and tool speed. In runs 1 and 2 , it takes 68.58 plate revolutions to accomplish the downward tool movement while in run 3 the plate revolves 137.16 times. Runs 1 and 3 both require 22.86 seconds to complete the tool movement while run 2 takes just half that time, 11.43 seconds. Clearly, the number of plate revolutions is more important to springback than the time required to complete the deformation. The effect of deformation time is seen between runs 1 and 2, and as one would expect from strain-rate hardening effects, the higher deformation rates result in greater amounts of springback, i.e. less permanent deformation. Again, the number of plate revolutions is most important.

Another important aspect of the tool feed rates is the amount of thinning that occurs in the part. It has been observed that the number of rotations also dominates this behavior with more rotations increasing the amount of thinning. While the detailed measurements of thinning were not made, it is visually evident and the simulations results to be presented shortly also demonstrate this effect.

Thermal measurements were made on the flat plate using a contact thermocouple. For a run 2 experiment, the starting plate temperature was $21.7 \mathrm{C}$ and at the end of the spin-forming (about 15 seconds after the completion of deformation) the plate rim temperature was $24.5 \mathrm{C}$ and temperature under the spinning tools was $37.3 \mathrm{C}$.

A photograph of a plate after deformation is given in Figure 2. The shiny disk below the plate is a standard computer $C D$ for a scale reference. Visible on the plate is a circular groove which marks the location where the spinning tools pressed the plate downward. There are two distinct marks in this groove, one for the 1 inch rim radius tool and another for the $3 / 8$ inch rim tool. 


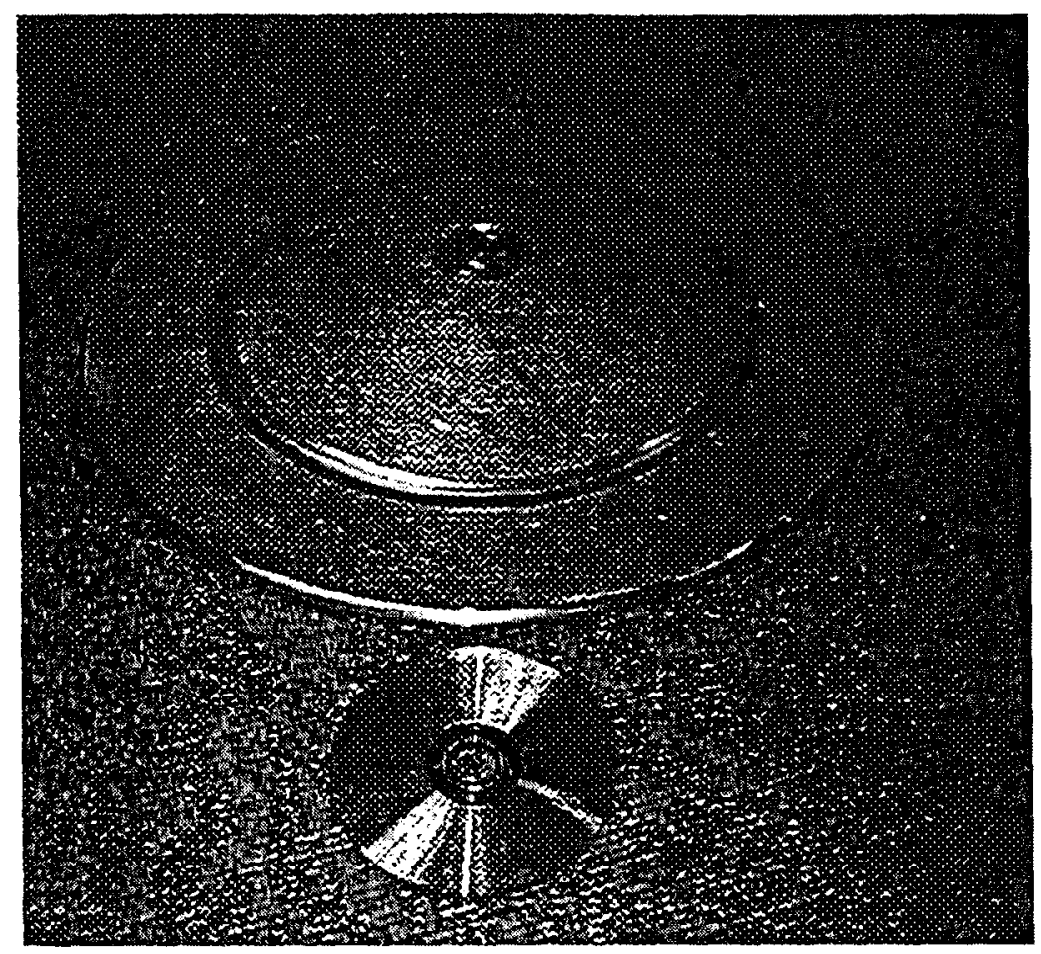

Figure 2 Spin-formed flat plate next to 5 inch $C D$

\section{Flat Plate Spin-Forming Simulations}

The simulation objectives in this LDRD are to test a variety of models, simulation methodologies and computer codes for their speed and accuracy in order develop a spin-forming simulation strategy. Specifically, the models employed in this work used solid elements, shell elements and rigid body formulations to represent tooling surfaces. For methodologies, two and three dimensional assumptions were tested. Simulations were performed where the part spun and the tools remained fixed and vice-versa. Static, dynamic and combinations of both were also tried. To speed-up the calculations tool velocities were scaled up, the number of rotations to complete the forming were varied, mass densities were altered and artificial damping introduced to improve the simulation fidelity. Four analysis codes were proposed for testing, NIKE3D [1], DYNA3D [2], ALE3D and DEFORM [4].

During all of these experimental tries at spin-forming simulations, the measures of acceptability were speed and accuracy benchmarks. The early first cuts at spin-forming simulations indicated that it would require thousands of hours of Cray CPU time to complete the simplest problems. For convenience, a useful measurement of the simulation speed is the compute time measure of CPU min/Part revolution. In other words, how long does it take the computer to model a single revolution of the spinning part. First measurements were at about $10 \mathrm{CPU} \mathrm{Hrs} / \mathrm{Part}$ revolution. In a typical spinning operation which lasts between 10 and 30 seconds, the spun part revolves at about 6 revolutions/second for a total of 60 to 180 revolutions; thus 100 revolutions would take $1000 \mathrm{Hrs}$.

The second benchmark is accuracy and that is taken from comparisons to part measurements and the ability to predict feed rate effects. In all these activities, it is necessary to balance simulation speed against accuracy and find the optimal compromise. During the course of this research, computing speeds improved by a factor of 10 or more. This definitely makes some calculations feasible, but these simulations still require significant CPU speedups to become truly practical, accurate and valuable to the spin-forming process developer who must form parts in a few days. The process development cycle now takes place in a few days of trial and error experimentation.

\subsection{Two Dimensional modeling}


Prior to this LDRD, most calculations in support of spin-forming were done in two dimensions using an axisymmetric assumption (Figure 3), and NIKE2D [4] was the analysis code used. This method is quiet useful for verifying tool paths and estimating the magnitude tool pass deformations. This last result is important for aiding the process developer in decisions concerning when to anneal a work piece in order to relieve it of accumulated residual stresses. The obvious drawback to this method is the error in the geometric assumption. The tool shape is wrong, the work piece is not undergoing cyclic stressing and the resulting deformation is independent of tool feed rates. An accuracy check against some hemispherical part shapes indicated an accuracy of $90 \%$ or better for predicting final part shape.

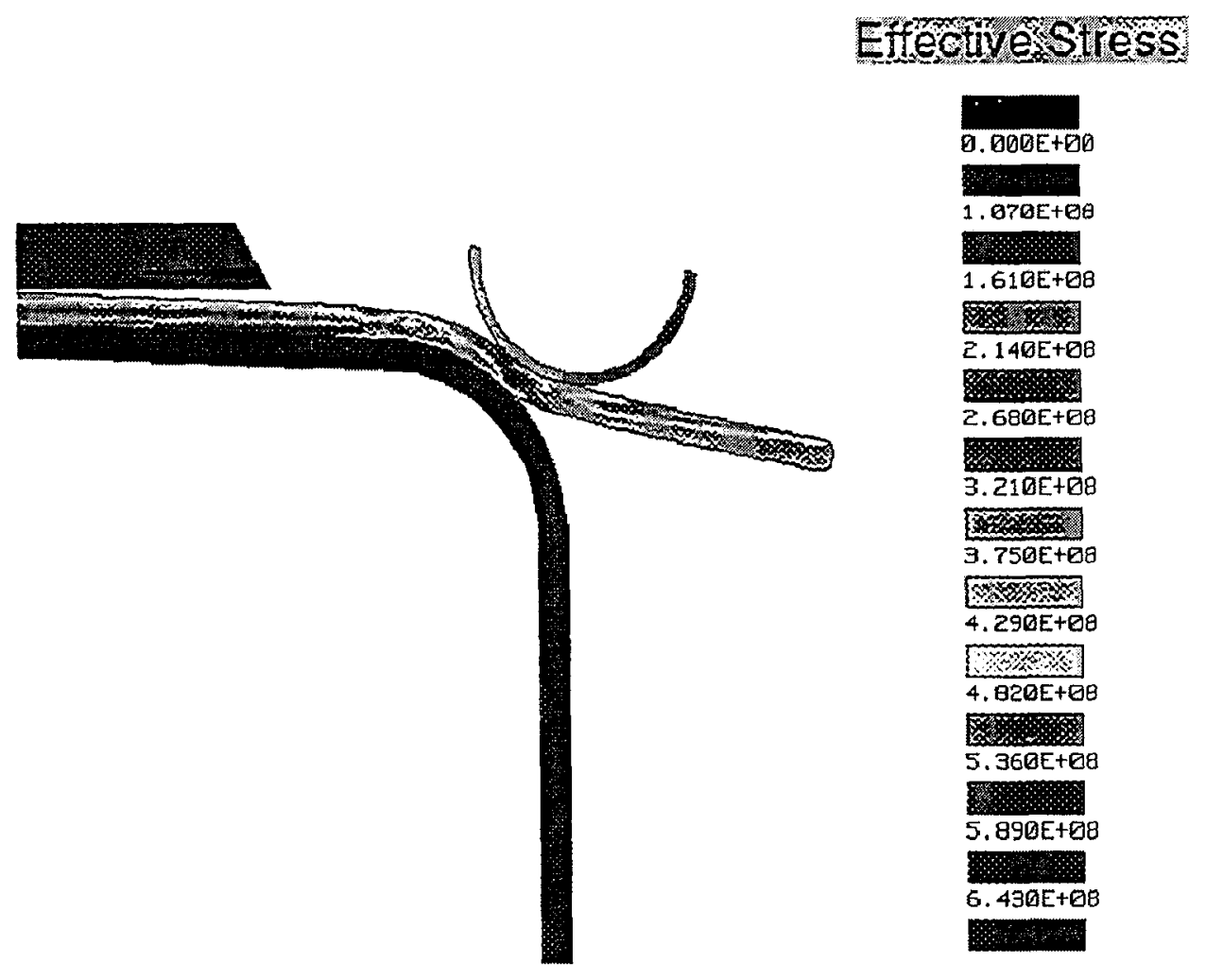

Figure 3 Axisymmetric spin-forming analysis

\subsection{Three Dimensional modeling}

Three dimensional analyses of spin-forming were performed using NIKE3D, DYNA3D and DEFORM3D. While calculations were proposed using ALE3D, the advice of ALE3D developers was that DYNA3D would be faster for this application and there were no unique features of ALE3D which differentiated it from DYNA3D.

DEFORM2D calculations were performed in axisymmetric mode and judged to be comparable to NIKE2D but easier to perform. In three dimensions, DEFORM3D was too difficult to use and/or it was just incapable of solving this problem. The effort of the DEFORM analyst was terminated early in this project when it became clear that the time investment would be significantly larger than NIKE3D and DYNA3D efforts.

The finite element model used to model the flat plate experiments is shown in Figure 4. The tools and mandrel are modeled with 1680 rigid shell elements and the Tailstock has 320 deformable elements. This basic model is used for both the NIKE3D and DYNA3D calculations. In the NIKE3D simulation, the plate is spun and the tools execute a downward motion. For DYNA3D, the plate remains stationary and tools spin around and down. 


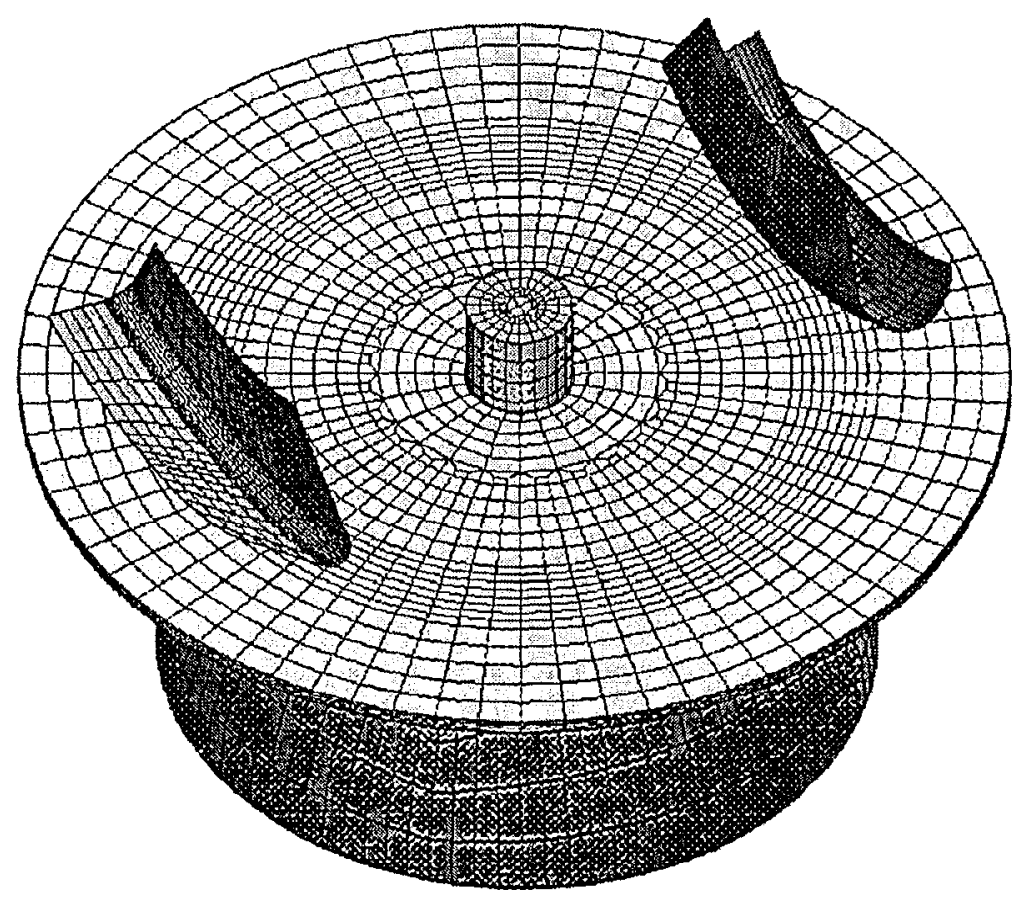

Figure 4 Finite element model of flat plate spin-forming

The finite element model of the flat plate is composed entirely of solid brick elements. Shell elements were examined for solving this problem, but a decision to not use shells was made for several reasons of accuracy. Predicting the grooved deformation of the plate requires many (5-10) elements across a groove that is approximately $1 / 2$ inch wide. The plate itself is $1 / 4$ inch thick. Thus, the required shell element resolution would result in element dimensions which are smaller in their thickness than in their plane dimensions, which is inappropriate. A similar argument which is just as cogent is that some of the plate deformation occurs in a shearing mode which is not adequately modeled with shells. The spin-forming deformations are not due to just a bending strains. The high degree of shear occurs in the groove and is not modeled well with shell elements. Thus, solid elements are the recommended modeling choice for spin-forming simulations.

The flat plate model is designed to give greater mesh resolution under the tools. Other aspects of the model attempt to create as realistic a simulation as possible. The Plate interfaces to the Mandrel and Tailstock are modeled as slide surfaces with friction $(\mu=0.1)$ and the Tailstock presses the plate to the Mandrel with a realistic pressure. The forming tools are modeled in 60 degree wedges, and they interface to the Plate in a frictionless slide surface mode. The Mandrel and Tool surfaces are modeled with many rigid shell elements to provide a smooth surface at low computational cost. The Tailstock is deformable, and its contact conditions with the Plate evolve as the Plate becomes curved and stresses redistribute in this area.

The number of elements through the thickness of the plate is a critical factor in the speed and accuracy of these finite element calculations. Under all circumstances, increasing the number of through thickness elements improves accuracy but at the expense of compute speed. With NIKE3D, the penalty from increased mesh size is not so critical, so more elements are advisable with the primary constraint is the deteriorating element aspect ratios. For DYNA3D, the penalty is much more severe because the plate thickness divided by the number of elements is the critical dimension for the Courant stability limit which determines the time step size.

There is a greater impact for DYNA3D when increasing the number of elements through the plate thickness. For a more equal comparison that leads to comparable compute speeds, the NIKE3D model uses 2 through thickness elements for a total plate element count of 3248 elements. DYNA3D models have 3 through thickness elements for a total of 4872 elements in the flat plate. Across the thickness, that gives NIKE3D 4 integration points and 3 nodes and DYNA3D 3 integration points and 4 nodes. 


\subsection{NIKE3D}

Spin-forming calculations with NIKE3D were performed to explore three areas of interest to these simulations: static versus dynamic simulations, implicit versus explicit finite element techniques and the impact on simulation accuracy and speed.

From a computational standpoint, there are several interesting conclusions to report (Table 2) concerning the performance NIKE3D. These calculations were completed in Sept. 1996 using a Dec Alpha SMP computer with $450 \mathrm{MHz}$ processors. In the NIKE3D analysis, the plate is stationary and the tools rotate around the plate. A plate rotation of $6 \mathrm{rev} / \mathrm{sec}$ results in a maximum centrifugal stress of just $0.03 \mathrm{psi}$, so these effects are insignificant. For a quasi-static analysis, the time step must takes a certain amount of plate rotation per step. This becomes the analysis parameter to investigate. Taking larger angular steps has an obvious advantage of requiring fewer steps to complete a tool rotation and the compute time data indicates the rapid decrease in compute time. However, these steps of ever larger angular rotation are much more difficult for NIKE3D to resolve in the contact algorithms. This increasing difficulty is demonstrated in the relative compute time numbers. It should be noted that for the larger angular step sizes, the tools are moving an entire element length per time step. Thus, the conclusion from this examination is that angular step sizes should be dictated by angular element dimensions and maximized to the greatest extent allowed by the contact algorithms. 


\begin{tabular}{|c|c|c|}
\hline $\begin{array}{c}\text { Angular Step } \\
\text { Size (degree) }\end{array}$ & $\begin{array}{c}\text { Compute Time } \\
\text { (CPU min/rev) }\end{array}$ & $\begin{array}{c}\text { Relative Compute Time } \\
\text { (CPU min/degree) }\end{array}$ \\
\hline 1 & 430.5 & 1.20 \\
\hline 7 & 83.2 & 1.62 \\
\hline 9 & 71.5 & 1.79 \\
\hline 10 & 70.7 & 1.96 \\
\hline 11 & 63.0 & 1.93 \\
\hline 12 & 56.7 & 1.90 \\
\hline
\end{tabular}

Table 2. NIKE3D computing time showing effect of angular step size on compute time and relative performance per angular degree.

The Table 2 calculations were performed using a quasi-static analysis assumption. A dynamic calculation repeating the 9 degree step size resulted in a compute time of $88.1 \mathrm{CPU} \mathrm{min} / \mathrm{rev}$ or a $25 \%$ decrease in compute speed. The resulting stresses did not change significantly. Shortly, after these results were completed, a new solver was added to NIKE3D which significantly reduced the required storage and factorization times. A repeat of the 9 degree step size calculation resulted in an decreased compute time of $31.5 \mathrm{CPU} \mathrm{min} / \mathrm{rev}$. The NIKE3D calculations were very successful in that the results were often very similar indicating some independence to angular step size. Thus the advantages to NIKE3D are more confidence in the results and a the ability to alter the step size and fine tune the compute time and accuracy.

\subsection{DYNA3D}

Computational simulations with DYNA3D require the application of special techniques to model the long forming times of 10-30 seconds, (relative to morc typical DYNA3D simulations). From NIKE3D experience, inertia and rotation effects are not important to spin-forming. So these conditions are exploited by increasing the rotation and tool fecd ratcs to shorten the time necessary to complete the metal forming and increasing the part densities to increase the time step size that DYNA3D would take to solve the problem.

The special DYNA3D techniques for a computational speed-up use mass density scaling and rotation rate increases. To accomplish this, the tools rotate and the plate remains stationary. Otherwise, rotational inertia effects seriously affect the plate stresses. For quasi-static analyses in NIKE3D, there are no dynamic effects so its tools can remain stationary and the plate rotates. NIKE3D simulations were performed in both modes.

For the DYNA3D flat plate simulations, the tool feed and rotation rates were simultaneously increased up to a maximum of 15 times faster than normal. The mass density scaling was increased by factors ranging up to 1000 . Initially, the accuracy of these results were seriously questioned. There was significant chatter between the plate and the forming tools. The chatter was due to rapid oscillations in the contact conditions between the plate and tools. These conditions created a non deterministic condition in the results because the solutions were far from any single converged result. Although the compute times were impressive, down to $17 \mathrm{~min} / \mathrm{rev}$ on the same 450 $\mathrm{MHz}$ Dec Alpha computer, the results were not consistent or in agreement with those from NIKE3D.

The problems posed by contact chatter were resolved by introducing damping to the DYNA3D solution. This led to results consistent with those from NIKE3D. Figures $5 \mathrm{a}$ and $5 \mathrm{~b}$ show the out of plane motion of several points on the surface of a plate in the path of the tools. The results from Figure 5a are undamped and clearly indicate the large chaotic like motion of the plate as it chatters. Figure $5 \mathrm{~b}$ shows the result for a $40 \mathrm{~Hz}$ critical damped analysis. The results are much smoother and compare reasonably to NIKE3D. The magnitude of the plate oscillations is affected by the amount of mass density scaling. These results are for a mass scale-up of 1000 which does lead to unwanted inertial damping or a lack of elastic spring-back during plate rotation. Decreasing the mass scaling increases the spring-back which is more realistic. 


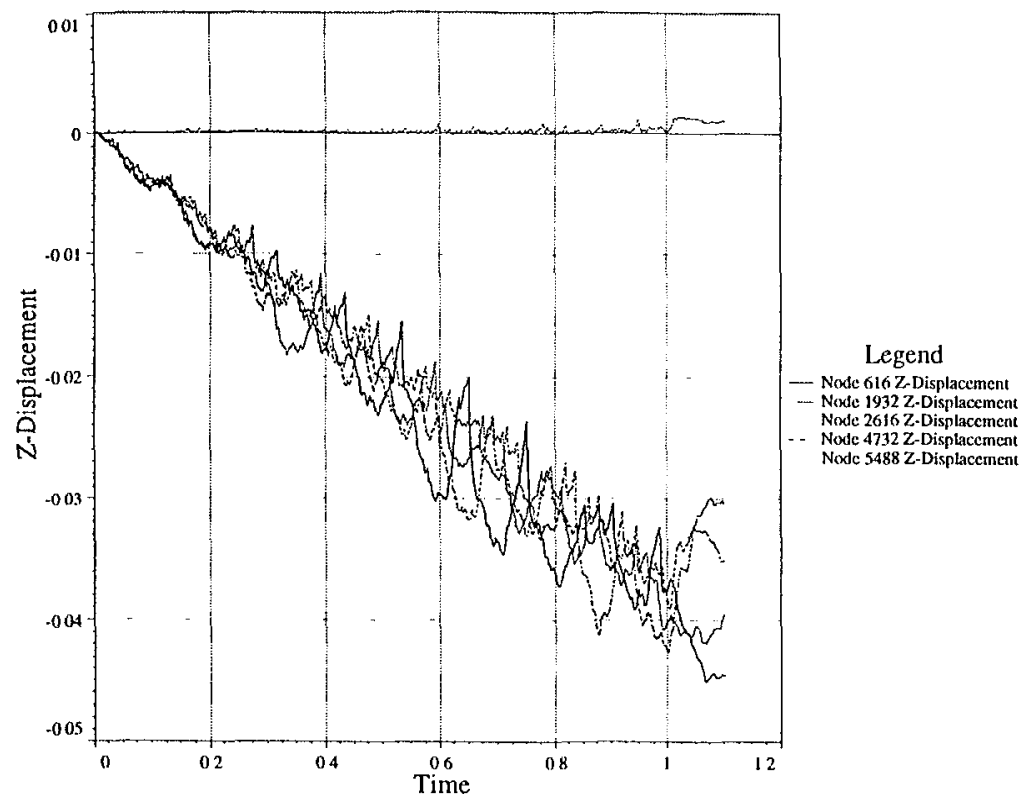

Figure 5a Undamped vertical displacement of points on the top surface of the flat plate under the tool path.

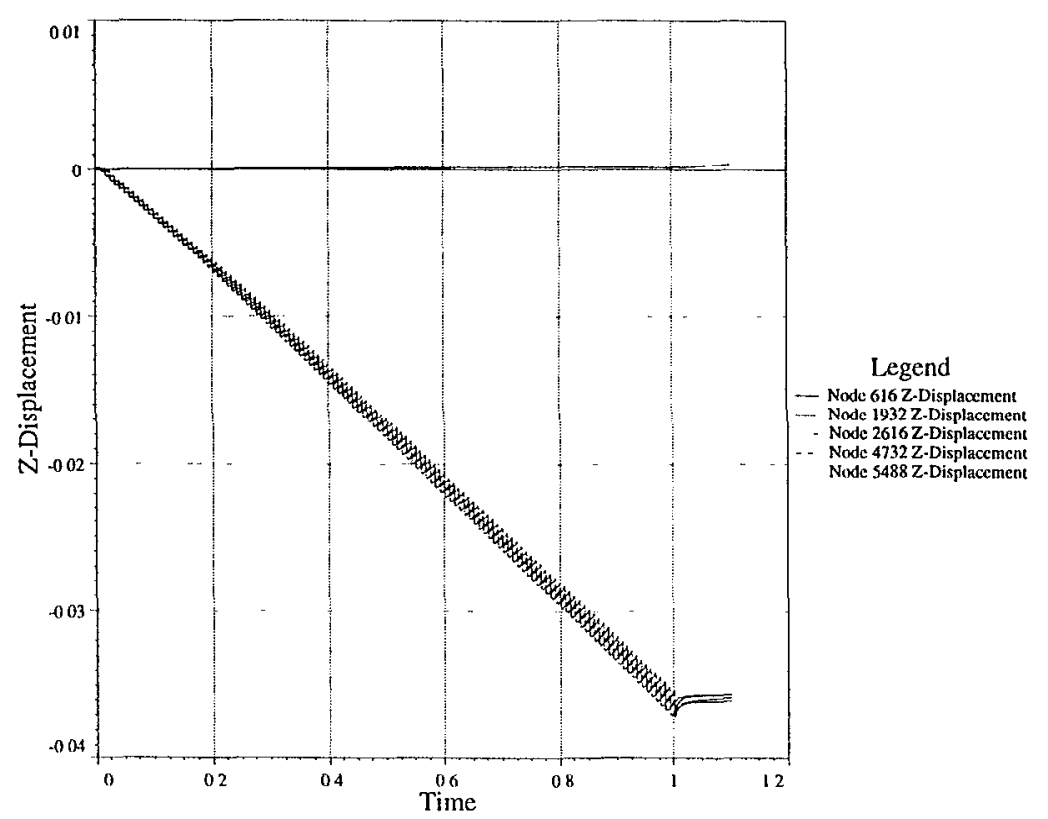

Figure $5 \mathrm{~b} 40 \mathrm{~Hz}$ damped vertical displacement of points on the top surface of the flat plate under the tool path.

A timing study was conducted for a rotation and tool feed rate speed-up of 5.0 with a critical damping of $40 \mathrm{~Hz}$. The compute times are shown in Table 3 . The 100 mass scaling and $5 x$ forming speed-up was the best compromise found in this work. Better compute times under-estimated the springback and are less accurate due to inertia errors. Slower compute times were more accurate but are less practical. It must be remembered that the 
effect of tool feed rates on forming deformation is most sensitive to the number of rotations completed. Thus, the compute time is paramount to making these predictions. The rate of deformation is less significant and since the material model used in these calculations is not rate dependent, these simulations do not capture these effects.

\begin{tabular}{|c|c|c|}
\hline $\begin{array}{c}\text { Simulation } \\
\text { Name }\end{array}$ & Mass Density Scaling & $\begin{array}{c}\text { Compute Time } \\
\text { (CPU min/rev) }\end{array}$ \\
\hline $\mathrm{sp} 053$ & 1.0 & 573.0 \\
\hline $\mathrm{sp} 153$ & 10.0 & 192.6 \\
\hline $\mathrm{sp} 253$ & 100.0 & 58.0 \\
\hline $\mathrm{sp} 353$ & 1000.0 & 17.0 \\
\hline
\end{tabular}

Table 3 DYNA3D compute time performance versus mass scaling.

\subsection{ParaDyn}

A parallel version of DYNA3D called ParaDyn was tested on a shortened run of the sp253 simulation to investigate the scaled speed-up of compute time with additional parallel processors. Since the finite element model size for spin-forming is quite small (4872 elements), and the contact conditions dominate the calculations, it was anticipated that processor scaling would be limited. These calculations were used as a benchmark problem by ParaDyn project leader by C. Hoover to test parallel contact algorithms. Table 4 indicates that using up to 8 processors can decrease the compute time from 58.0 down to $16.5 \mathrm{CPU} \mathrm{min} / \mathrm{rev}$.

\begin{tabular}{|c|c|c|}
\hline $\begin{array}{c}\text { Number of } \\
\text { Processors }\end{array}$ & $\begin{array}{c}\text { Compute Time } \\
\text { (CPU min/rev) }\end{array}$ & $\begin{array}{c}\text { Parallel } \\
\text { efficiency \% }\end{array}$ \\
\hline 1 & 58.0 & \\
\hline 2 & 32.5 & 89.3 \\
\hline 4 & 22.6 & 64.2 \\
\hline 8 & 16.5 & 44.0 \\
\hline
\end{tabular}

Table 4 Parallel compute time and efficiency for sp253 on Dec Alpha

Figure 6 shows how the increase in processor count speeds the calculations up, and it also shows how the parallel efficiency drops off as individual processors are not utilized effectively due to excessive inter-processor communication. Increasing the number of elements in these simulations does not seriously affect the compute speed so long as the minimum time step size is not decreased. More in-plane elements can be accommodated with more processors, but decreasing the time step size will slow down the compute time no matter how many processors are used. 


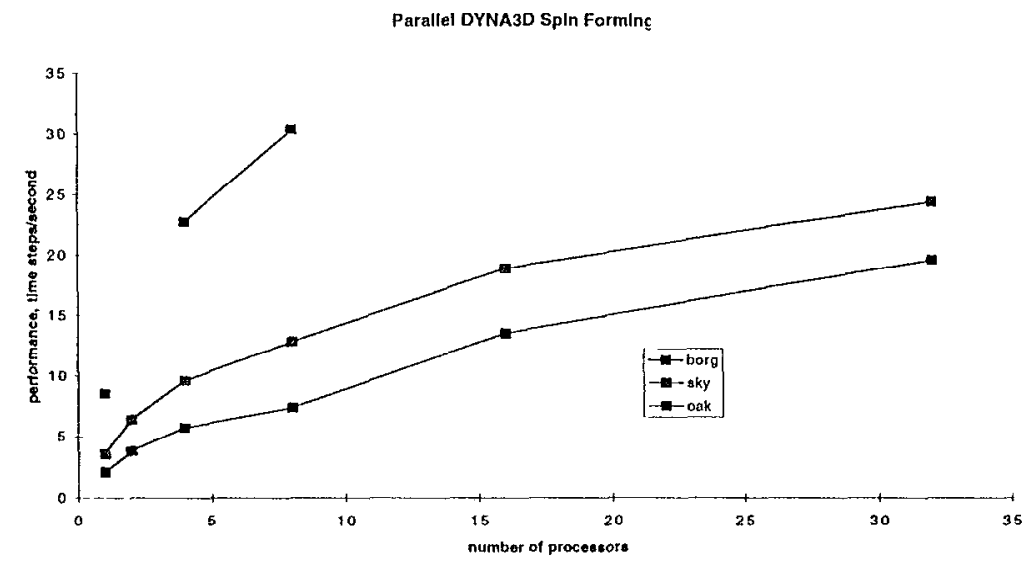

Figure 6 ParaDyn performance curves for flat plate spin-forming.

\subsection{Simulation Results}

Since the purpose of this work was to explore practical simulation strategies, less attention is paid to specific results and the emphasis is placed on compute time and consistent, realistic results. This scction will show a typical set of spin-forming predictions and conclude with comments summarizing the practicality of using flat spin-forming simulations.

The point has already been made several times that the number of spinning rotations in a forming operation has a significant effect on the final deformation. Since increasing the rotations leads to less springback it would seem obvious to simply increase this process parameter. Figure 7 shows the stress response at 3 locations in a short 6.25 rotation NIKE3D spin-forming simulation. The blue curve is for the plate top center. The red curve for the top surface of the plate at the tool radius. The green curve is at the outer top radius of the plate. The critical conclusion from this prediction is that spin-forming is a low cycle fatigue process. The number of plate rotations times two equals the fatigue cycles for the forming operation. Thus, the number of fatigue cycles must be minimized to limit damaging crack growth. 


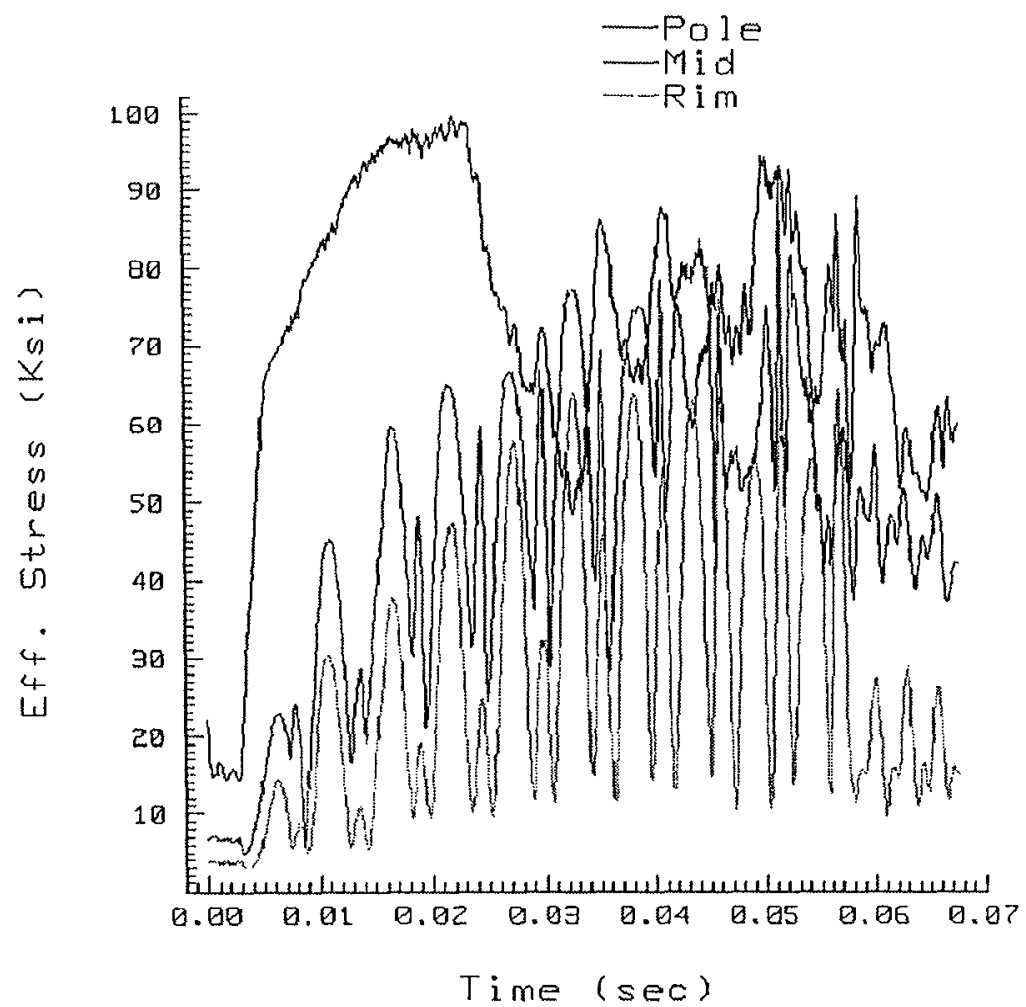

Figure 7 Cyclic stress response in a NIKE3D flat plate spin-forming simulation

Figure 8 shows a residual stress pattern at the end of the sp353 simulation. In Figure 2, a groove can just be barely be seen in the top plate surface. Much of the residual stress is confined to the grooved region on the top surface of the plate. There is an asymmetry to the residual stress that is due to an insufficient number of plate rotations. Thus, realistic simulation must have the proper number of plate revolutions. This DYNA3D simulation, with a factor of 1000 in mass density scaling, over-predicts deformation along the tool groove. Figure 9 shows the predicted plate cross section, with the vertical axis scaled by two. There are two outlines in this figure, one for the plate at the maximum tool depth and the other after springback. The closest experiment to this simulation had a predicted part height of $37.0 \mathrm{~mm}$. The predicted height is $41.7 \mathrm{~mm}$. The predicted dihedral angle of the simulation is in fact present in the spin-formed flat plates.

Decreasing the mass scaling narrows the difference between the simulation results and the actual measured height. Figures $10 \mathrm{a}$ and $10 \mathrm{~b}$ show the final effective plastic strain distributions where the difference in these results is due to mass scaling effects alone: 100 and 1000 times the mass density respectively. There is nearly twice as much deformation for the higher mass density scaling. No attempt was made to bring the simulation answers into agreement, because there are still too many factors affecting these results for that adjusting process to be meaningful. Decreasing the mass scaling increases the compute time and a realistic simulation should have the proper number of plate revolutions: about 90 . 


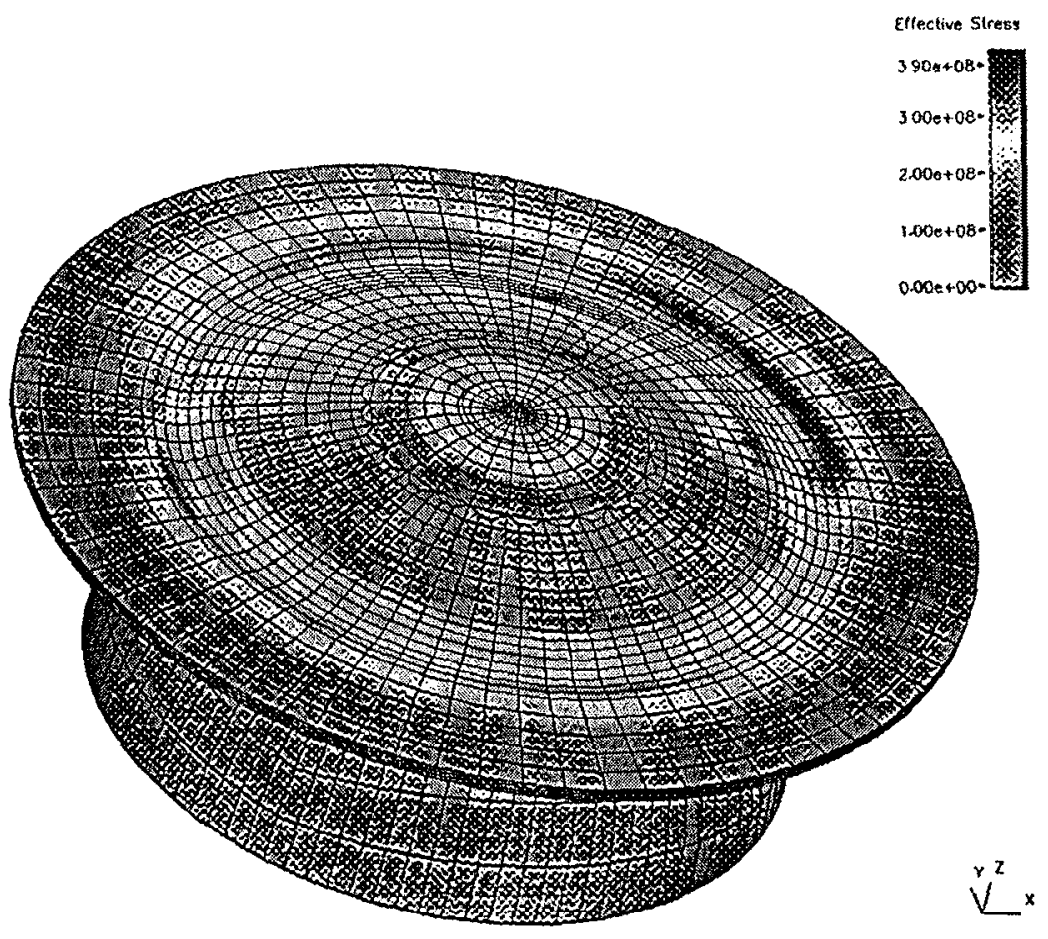

Figure 8 residual effective stress $(\mathrm{Pa})$ pattern at conclusion of sp253 DYNA3D simulation

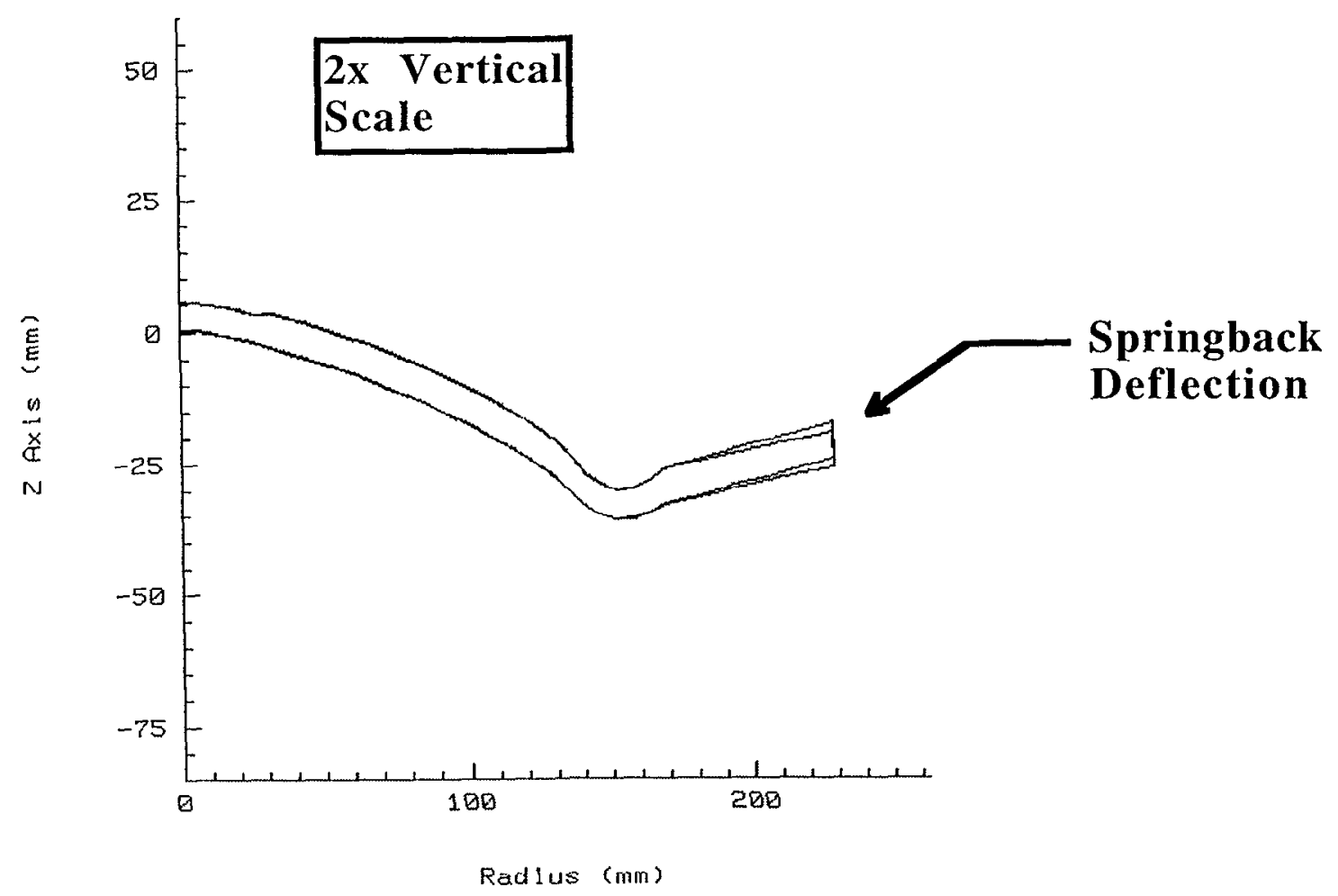

Figure 9 Springback in sp353 DYNA3D simulation. 


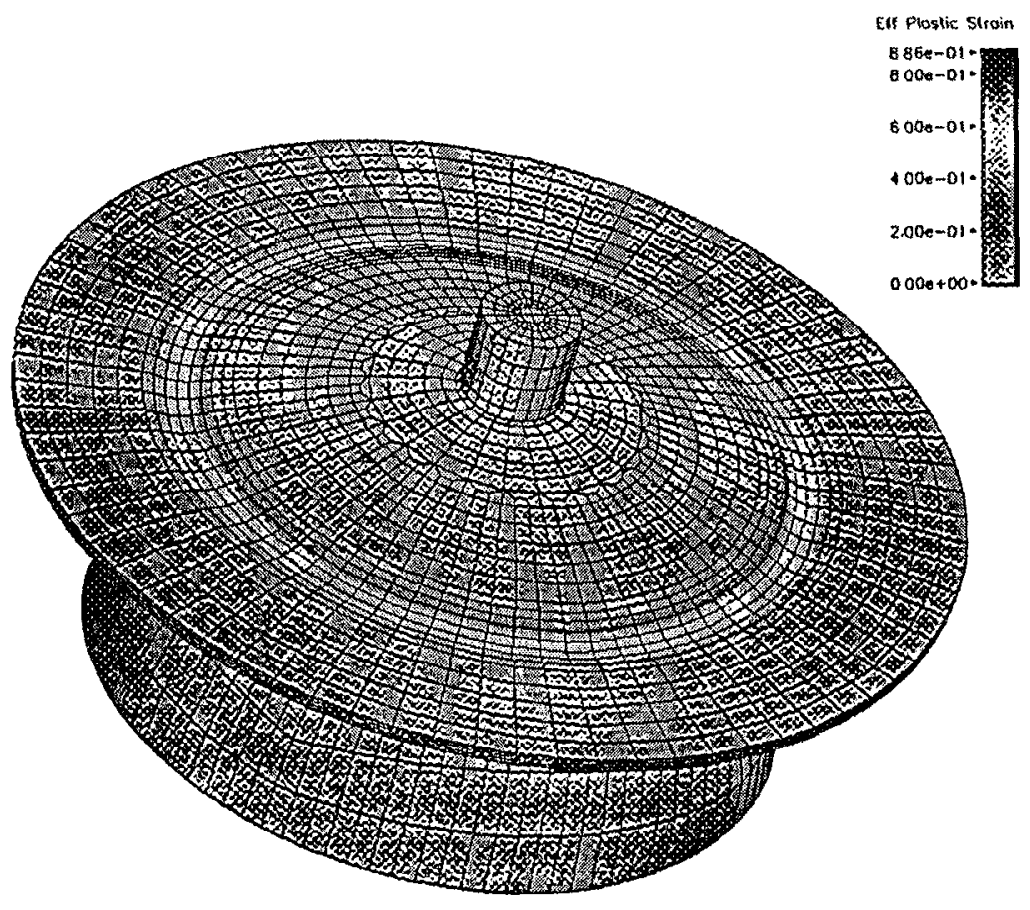

Figure 10a Final effective plastic strain for sp253 DYNA3D simulation.

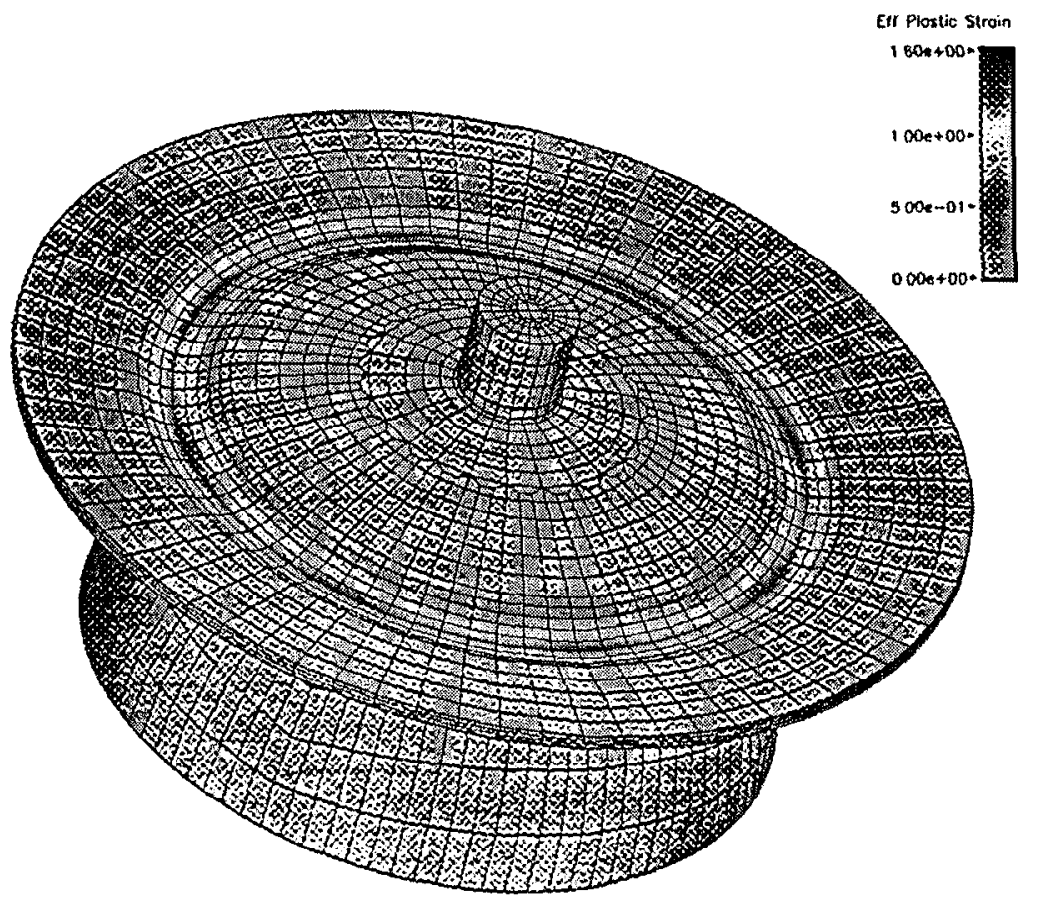

Figure 10b Final effective plastic strain for sp253 DYNA3D simulation. 
The simulations with NIKE3D took twice as long as DYNA3D, 31.5 versus $16.5 \mathrm{CPU}$ min/rev. But NIKE3D had several advantages which makes it a better choice for spin-forming simulations. First and foremost is the implicit advantage of large time steps. Second, without dynamic effects the results were more consistent, increasing the simulation confidence. Lastly, more elements can be placed through the plate thickness without a severe penalty to the compute speed. The big disadvantage to NIKE3D is its sensitivity and difficulty in achieving converged simulation results. The big advantage to DYNA3D is its reliability in providing results. The DYNA3D difficulty comes in verifying their veracity.

Practical spin-forming simulations will become commonplace when the compute times decrease to $8 \mathrm{CPU}$ $\mathrm{min} / \mathrm{rev}$. and less. For a typical spinning operation which lasts about $15 \mathrm{~seconds}$ at $60 \mathrm{rev} / \mathrm{sec}$, a compute time of $8 \mathrm{CPU} \mathrm{min} / \mathrm{rev}$ leads to a simulation duration of $12 \mathrm{CPU}$ Hrs. The typical spun part requires 6 or more spinning passes or operations. Thus, in order to model the entire part forming process in a 12 Hrs, a compute time of 1 CPU $\mathrm{min} / \mathrm{rev}$ is required. So a realistic simulation today requires more than 2 weeks of continuous calculations to model the entire forming operation. Faster computers will be the key, and then spin-forming modeling offers the routine opportunity to understand a very complex deformation process.

\section{Cylindrical Spin-Forming}

Cylindrical spin-forming simulations were performed by A. Lee to ascertain the feasibility of this metal forming method and to estimate the computational time of these simulations. To date, there have been no cylindrical spinforming operations or experiments at LLNL, so it is appropriate to calculate some numerical predictions for the process. As in the case of flat plate spin-forming, the determination of compute time is the important measure of the practicality of spin-forming computations.

For cylindrical simulations (Figure 11), a cylindrical blank is reduced in diameter to examine the effects of ovaling, buckling and mandrel loads. The model consists of 4 parts: Mandrel, Blank and the previous two Spinning Tools. The Mandrel and Tools are both modeled as rigid materials. Solid elements are used to model the Mandrel and Blank, and shell elements represent the Tools. There are 17119 nodes, 10560 solid elements and 720 shell elements. In order to minimize the analysis time, the Blank has only three elements through the thickness of 0.1 inches. This is thinner than the flat plate thickness of 0.25 inches and significantly contributes to a higher compute time. Several different boundary conditions were applied to constrain the Blank to the Mandrel and the results from these simulations suggest that the constraining loads can be significant.

The tool motion for cylindrical spin-forming is more complicated than for flat plate forming because the tools have a radial motion component. For cylindrical and hemispherical forming the true tool motions are axial and radial while the plate motion is angular. For simulation purposes, these relative motions can be shared between the parts. For the cylindrical spin-forming, a true realistic approach was taken with the Blank and Mandrel spinning and the Tools moving axially and radially relative to the spinning axis. The earlier flat plate spinning assigned the plate rotation to the tool, but combining that approach with the relative Tool motion results in very complicated tool motion paths that are tedious to create. The drawback to not pursuing this approach is that the benefits of mass density scaling and rotation speed-ups are diminished due to concerns with rotational inertia stresses. 


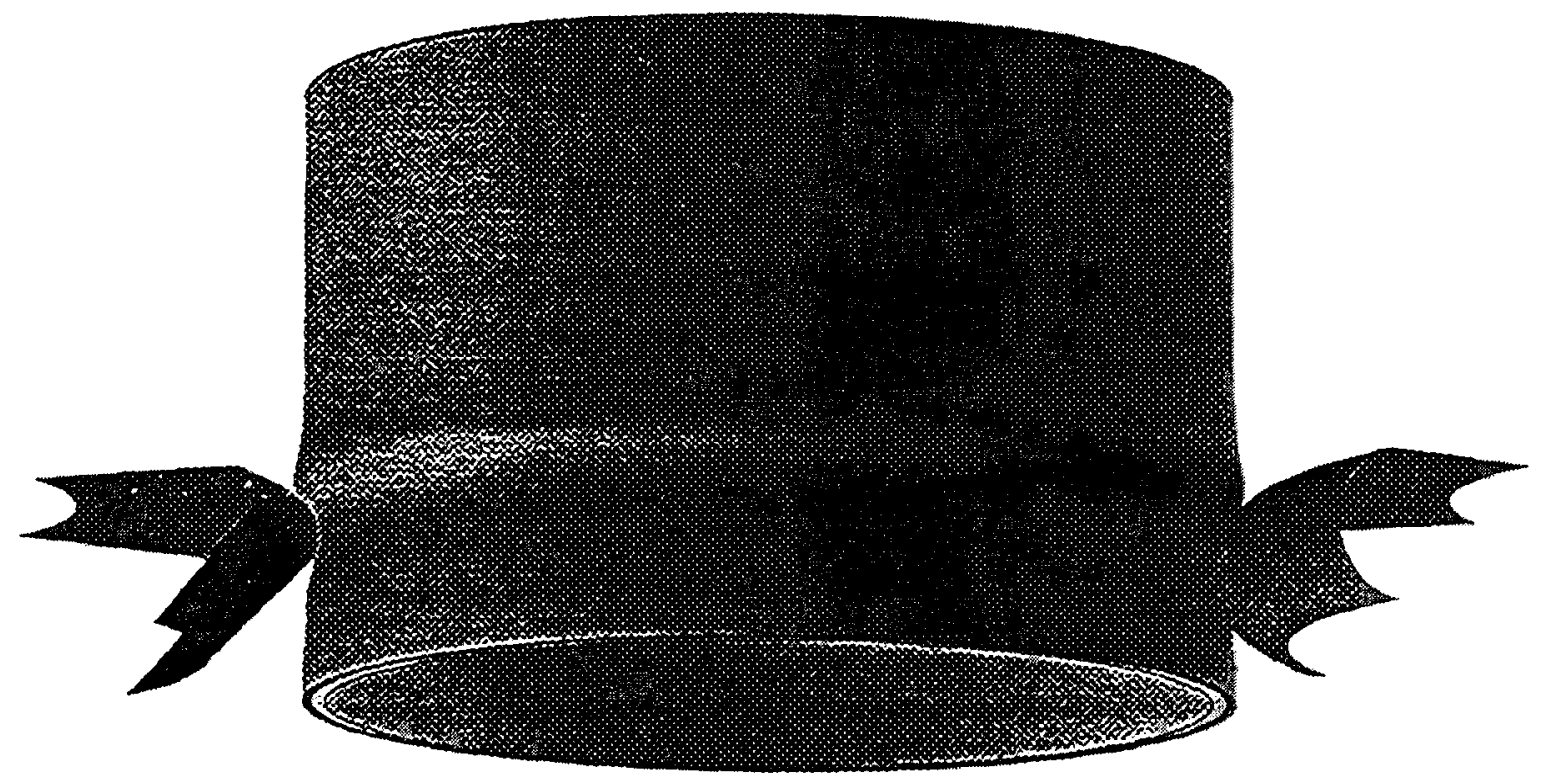

Figure 11 Cylindrical spin-forming simulation

\subsection{Single sliding interface approach}

Table 5 summaries the cylindrical spin-forming analyses performed for this study. Four analysis parameters were being varied in the study: mass scaling, feed rate $V_{f}$, hourglass stabilization method IHQ, and mass proportional damping. The model used a single sliding interface for each individual component: one sliding interface for the entire blank, another sliding interface for the entire mandrel, and two more sliding interfaces for the spinning tools. A Lagrangian constraint contact method was used as it performed better than the default penalty method.

\begin{tabular}{|c|l|l|}
\hline feed rate & \multicolumn{1}{|c|}{ Mass } & \multicolumn{1}{c|}{ 2 X Mass } \\
\hline $\mathrm{V}_{f}$ & $\mathrm{IHQ}=$ viscous form & $\mathrm{IHQ}=$ viscous form \\
\hline $1.5 \mathrm{XV}_{\mathrm{f}}$ & $\begin{array}{l}\mathrm{IHQ}=\text { stiffness form } \\
\text { (exact volume) }\end{array}$ & $\mathrm{IHQ}=$ stiffness form, 50 Hz damping \\
\hline & & $\mathrm{IHQ}=$ stiffness form \\
\hline & & $\mathrm{IHQ}=$ stiffness form, $50 \mathrm{~Hz}$ damping \\
\hline
\end{tabular}

Table 5 Cylindrical spin-forming analysis list.

Note that the fecd rate $V_{f}$ used in the analysis was $7.5 \mathrm{inch} / \mathrm{sec}$ which is 15 times higher than the probable machine feed rate of $0.5 \mathrm{in} / \mathrm{sec}$. Similarly, the analysis rotation rate was $100 \mathrm{rev} / \mathrm{sec}$ which is 16.67 times faster than the actual machine rotation rate of $6 \mathrm{rev} / \mathrm{sec}$. The primary motive of mass scaling and increasing the feed rate and the rotation rate is to shorten the CPU time. Typical analysis on a Dec Alpha machine required 9 CPU Hrs/ rev.

Ovaling of the blank due to springback was evident in all the analyses except in those analyses that were using default (viscous form) hourglass stabilization method. Ovaling was also more pronounced with mass scaling. Mass proportional damping was included in some analyses in an attempt to minimize the ovaling effect but without much success.

The type of hourglass stabilization method governed how well the blank would conform to the mandrel and the overall forming behavior. Slight hourglassing in the blank was observed in those analyses where the default method was used. In these analyses, the blank conformed to the mandrel rather well except it "bunched" ahead of the spinning tools. On the other hand, using the stiffness form of hourglass stabilization method, hourglass modes 
were not observed but the blank sprang away from the mandrel when it was no longer in contact with the spinning tools. Moreover, the blank in these analyses was "twisted", meaning the elements at the lower end of the blank were lagging behind the upper end of the blank.

Deformation plots and additional information are given in another report [5].

\subsection{Multiple sliding interface approach}

Since the spinning tools are in contact with the same region of the blank only for a finite period of time, conceivably, multiple sliding interfaces could be defined for the blank and have only one sliding interface active at a time. Sliding interfaces can be activated or deactivated as the spinning tools pass over the blank. The potential benefit of having multiple sliding interfaces is to be able to partition these sliding interfaces into multiple processors for ParaDyn. Even though the current partitioning software could not partition Lagrangian constraint type sliding interfaces into different processors, it may be worthwhile to pursue this approach in the future when the partition software acquires new capabilities.

A preliminary analysis with multiple sliding interfaces was attempted with the serial DYNA3D. However, the deformation and results appeared to be wrong. Further investigation is required to identify the problem in the model.

Cylindrical spin-forming analyses point out that much more work is required before practical spin-forming simulations are feasible for cylindrical geometries. The compute time needs a factor reduction of more than 500 . Algorithmic issues such as sliding interfaces are important contributors to the speed-up. Other algorithm issues such as hourglass stabilization methods can be resolved with experimental data comparisons and greater experience with the expected and proper response of cylindrical spin-forming processing. Additionally, convenient methods to automatically prescribe tool motions that will allow greater mass density, cylinder rotation speeds and tool feed rates need to be developed. Based on the predicted results obtained from these simulations, cylindrical spin-forming will be far more difficult than flat plate spinning, so computer simulations may have greater importance.

\section{Shape-Memory Modeling}

An important spin-forming milestone in this LDRD project was the development of a constitutive material model for predicting the shape memory behavior U-Nb alloy. Based upon experimental data obtained for various alloy compositions, measurements of stress-strain response and phase compositions at various temperature and processing histories were obtained by Vandemeer [6]. A contract with Asst. Prof. S. Govindjee at the Univ. of Calif. at Berkeley was executed to develop a finite element theory to provide a basis for the development of a finite element material model. His finished report entitled "A Shape Memory Alloy Mode for Uranium-Niobium Accounting for Plasticity" [7] describes a 1-D model which predicts a bilinear isotropic plasticity model with shape memory effects that model three phase transformation processes. He also did a material property characterization to fit $\mathrm{U}-\mathrm{Nb}$ alloy data to the model parameters in his report.

Figure 12a shows the measured stress strain response in a thermo-mechanical experiment. The upper portion of the plot shows the stress strain response and the lower portion gives the temperature strain response which is imposed after the mechanical stress cycle. Two processing conditions are given in these plots. In the first, a mechanical loading stresses the material to about $7 \%$ strain and then unloads. This is followed by a thermal cycle between 298 and $1078 \mathrm{~K}$. The second loading sequence is out to about $17 \%$ strain followed by the same thermal cycle. A thermal cycle for $7 \%$ strain loading was completely reversible while going to $17 \%$ resulted in permanent deformation. Figure $12 \mathrm{~b}$ shows the predicted results from the material model developed for this LDRD. 


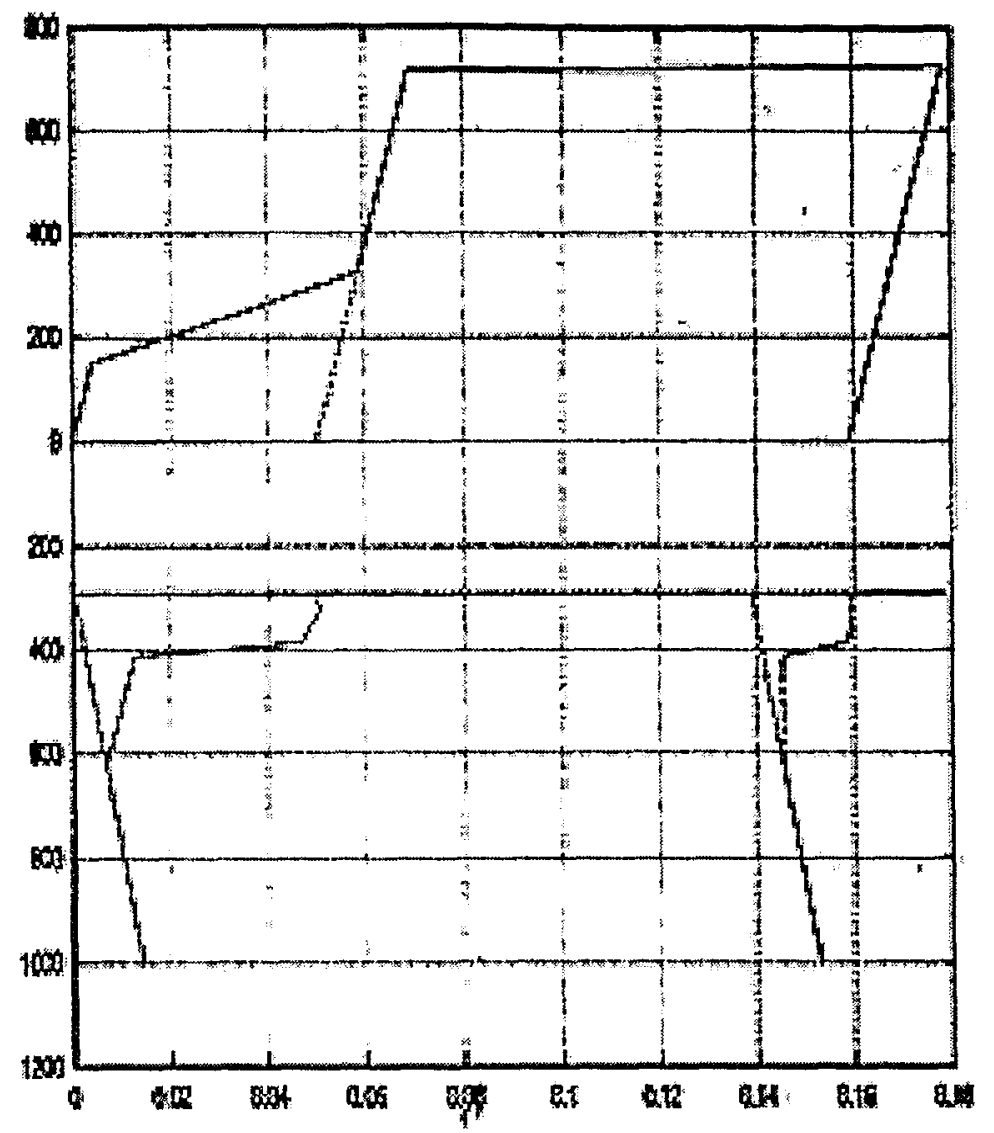

The 1-D model has been programmed into a model for NIKE2D, but the practicality of using this model in a fully 3-D scenario is premature. The 1-D experiments and material model do not adequately predict the three dimensional nature of shape-memory effects. Shape memory materials show compressive versus tensile history effects and given the oscillatory nature of spin-forming, a 3-D model development is still required.

Since U-Nb alloys are of interest to spin-forming, it is important to include their effects in these simulations. The "double-knee" stress strain curves is expected to change the deformation and springback response of these simulations. Furthermore, when these parts experience an annealing thermal cycle, material having deformed between $7-17 \%$ is expected to recover a portion of its mechanical strain and return to its undeformed shape. These shape memory effects have a significant effect on the development of spin-forming process parameters, and finite element simulations are seen as potential solution to an impractical trial and error method of process parameter development.

\section{Conclusions}

Practical spin-forming analysis methods, which aid process development, can simulate flat plate spin-forming for ordinary materials. Implicit code methods such as those employed by NIKE3D are more accurate, simpler in modeling realistic forming conditions, but slower than explicit methods such as DYNA3D. DYNA3D and especially ParaDyn give the fastest compute times, but require special attention and skill to obtain accurate solutions. Large compute speed improvements are still needed to simulate cylindrical spin-forming processes. 3D shape memory material models need to be developed for modeling spin-forming and annealing processes. Integrating computer aided design with computer aided manufacturing and computer simulations was achieved through SmartCAM software. Because an adaptive control system was not developed during the course of this work, an important component of a fully integrate computational simulation capability is yet to be realized. 


\section{REFERENCES}

[1] Maker , B.N., 'NIKE3D: User Manual', Rept. UCRI_-MA-105268, Lawrence Livermore National Laboratory, Livermore, CA, 1995.

[2] Whirley, R.G., 'DYNA3D: User Manual', Rept. UCRL-MA-107254, I awrence Livermore National Laboratory, Livermore, CA, 1993.

[3] Wei-Tsu Wu, 'DEFORM-3D V. 2.1 Manual', Scientific Forming Technologies Co., Columbus, OH 1996.

[4] Englemann., 'NIKE2D: User Manual', Rept. UCRL-MA-105413, Lawrence Livermore National Laboratory, Livermore, CA, 1991.

[5] A. Lee, "Cylindrical Spin-forming Analysis Notes", COMN-98-004, Lawrence Livermore National Laboratory, Livermore, CA, 1998

[6] Vandermeer, R.A., Ogle, J.C., and Northcutt, W.G., "A Phenomenological Study of the Shape Memory Effect in Polycrystalline Uranium-Niobium Alloys," Metallurgical Trans. A, 12A, 733-741 (1981).

[7] Govindjee, S., and Kasper E.P., "A Shape Memory Alloy Model for Uranium-Niobium Accounting for Plasticity”, Rept. UCB/SEMM-97/06, Dept. of Civil Eng., Univ. of Calif., Berkeley, CA, 1997. 


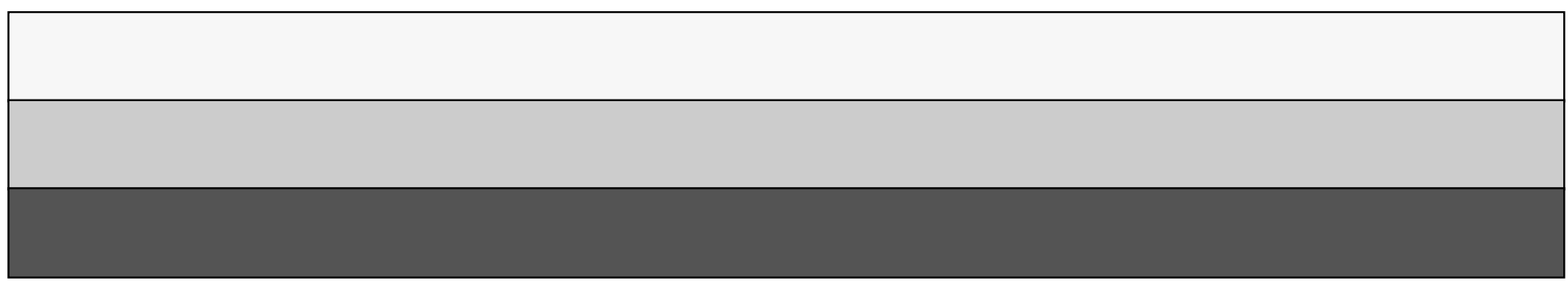

